UCRL-JC-

PREPRINT

CONF-9208276--1

\title{
Nonequilibrium Molecular Dynamics: The First 25 Years
}

\author{
William G. Hoover \\ University of California at Davis/Livermore \\ Lawrence Livermore National Laboratory \\ University of California \\ Livermore, CA 94551, USA
}

This paper was prepared for submittal to the proceedings of the

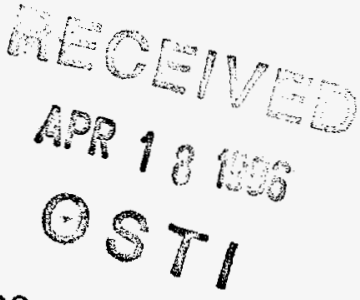

18th IUPAP International Conference on Statistical Physics

August 2-8, 1992

Berlin, Germany

August 1992

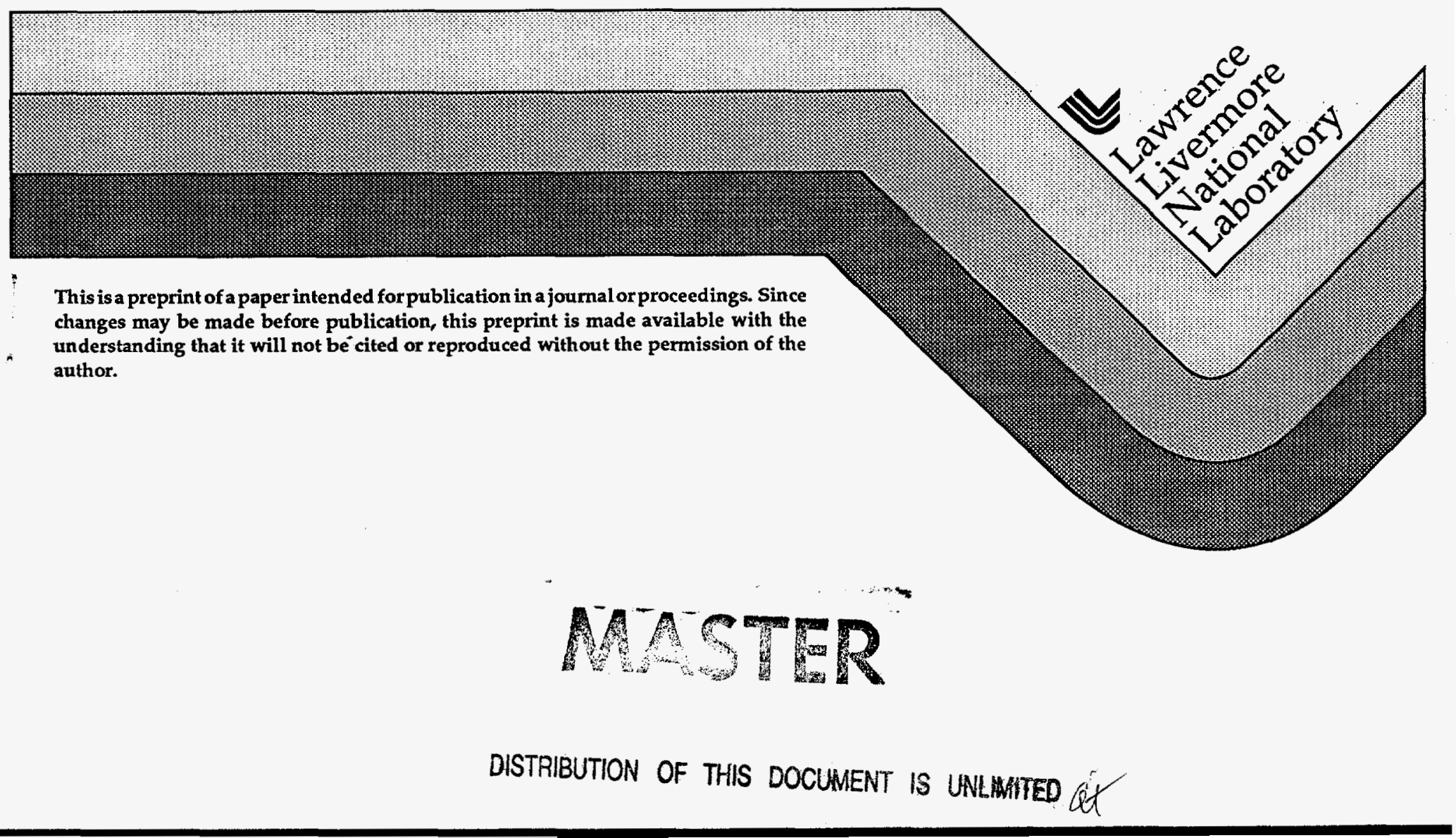




\section{DISCLAIMER}

This document was prepared as an account of work sponsored by an agency of the United States Government. Neither the United States Government nor the University of California nor any of their employees, makes any warranty, express or implied, or assumes any legal liability or responsibility for the accuracy, completeness, or usefulness of any information, apparatus, product, or process disclosed, or represents that its use would not infringe privately owned rights. Reference herein to any specific commercial products, process, or service by trade name, trademark, manufacturer, or otherwise, does not necessarily constitute or imply its endorsement, recommendation, or favoring by the United States Government or the University of California. The views and opinions of authors expressed herein do not necessarily state or reflect those of the United States Government or the University of California, and shall not be used for advertising or 


\section{DISCLAMMER}

Portions of this document may be illegible in electronic image products. Images are produced from the best available original document. 


\section{Running Title: 25 Years of Nonequilibrium Behavior}

Nonequilibrium Molecular Dynamics: The First 25 Years

\section{Wm G Hoover}

Department of Applied Science, University of California at Davis/Livermore,

Department of Physics, Lawrence Livermore National Laboratory,

Post Office Box 808, L-794, Livermore, California, 94551, United States of America.

\section{Abstract:}

Equilibrium Molecular Dynamics has been generalized to simulate Nonequilibrium systems by adding sources of thermodynamic heat and work. This generalization incorporates microscopic mechanical definitions of macroscopic thermodynamic and hydrodynamic variables, such as temperature and stress, and augments Atomistic forces with special Boundary, Constraint, and Driving forces capable of doing work on, and exchanging heat with, an otherwise Newtonian system:

$$
\left\{\dot{p} \equiv F_{A}(q)+F_{B}(q)+F_{C}(q, p)+F_{D}(q, p)\right\} \equiv m\left[q_{t+d t}-q_{t}+q_{t-d t} / d t^{2}\right\}
$$

The underlying Lyapunov instability of these nonequilibrium equations of motion links microscopic time-reversible deterministic trajectories to macroscopic time-irreversible hydrodynamic behavior as described by the Second Law of Thermodynamics.

Green-Kubo linear-response theory has been checked. Nonlinear plastic deformation, intense heat conduction, shockwave propagation, and nonequilibrium phase transformation have all been simulated. The nonequilibrium techniques, coupled with qualitative improvements in parallel computer hardware, are enabling simulations to approximate real-world microscale and nanoscale experiments. 


\section{Motivation/Goals:}

Three strong motivations led directly to nonequilibrium molecular dynamics: it furnished a welcome check for Green and Kubo's linearresponse theory of transport; it promised success in treating nonlinear problems; it furnished a new tool for understanding real phenomena. These goals parallel Zwanzig's reviews ${ }^{1}$ of the motivation underlying nonequilibrium statistical mechanics. In 1977, in a perceptive speech in Kyoto ${ }^{2}$, Kubo likewise discussed the prospects for theoretical physics. Pointing out that physicists are not afraid to shun formalism and to face facts, Kubo emphasized that nonlinear problems were the only problems left. To solve these problems, once computers became available, nonequilibrium molecular dynamics had to be developed. The development was carried out by many people 3 . It is natural that they had, and still have, similar ideas at about the same time.

\section{Relation to Thermodynamics and Hydrodynamics:}

A century ago, Lyapunov analyzed the dynamic stability of differential equations. Linear analysis of the growth of a trajectory perturbation, $\dot{\delta} \propto$ $\delta$, gives just three possibilities: decay, oscillation, and divergence. The last case-divergence and exponential "sensitivity to initial conditions" defines Lyapunoo instability. The biggest Lyapunov exponent $\lambda_{1}$ gives the average rate at which two neighboring trajectories diverge, $\delta(t) \approx$ $\delta(0) \exp \left(\lambda_{1} t\right)$. The rate at which the area of an ellipse, defined by three neighboring trajectories, diverges defines the next exponent, and so on: $\mathrm{A}(\mathrm{t}) \approx \mathrm{A}(0) \exp \left(\lambda_{1} t+\lambda_{2} t\right)$. Lyapunov's "instability chaos" is the fundamental link between reversible microscopic nonequilibrium dynamics and irreversible macroscopic physics. 
Lyapunov's ubiquitous exponential divergence underlies Boltzmann's 1872 concept of "molecular chaos", the random orientation of collision partners in a low-density gas. Boltzmann's equation in turn links microscopic molecular chaos to macroscopic irreversibility and transport properties. It was not until 1967 that Lorenz' analysis of weather forecasting popularized and underscored the importance of Lyapunov instability4. Now, the "Butterfly Effect", "chaos" and "Lyapunov instability" are familiar parts of our physics vocabulary 5 and key ingredients in our understanding of nonequilibrium molecular dynamics.

Both temperature and thermostats are missing in Newtonian mechanics. Both are required to simulate the energy flows described by the thermodynamics and hydrodynamics of nonequilibrium systems. Kinetic theory furnishes the operational definition of temperature through the ideal-gas thermometer. In nonequilibrium molecular dynamics temperature is always measured by kinetic energy.

Theoretical analysis is greatly simplified if the generalized constraint and driving forces of nonequilibrium molecular dynamics are deterministic and time-reversible. The simplest such thermostat can be based on Gauss' Principle of Least Constraint. The corresponding constraint forces keep the kinetic energy of a selected set of degrees of freedom constant 6 . A more elegant alternative Gibbsian constraint force, producing the canonical distribution rather than the isokinetic one, was discovered by Nosé in 19847, and was recently generalized by Bauer, Bulgac, and Kusnezov8.

We will see that Gibbs' definition of entropy, $S_{\text {eq }} \equiv-k<\operatorname{lnf}>$, and the corresponding equilibrium definition of temperature, $T \equiv(\partial E / \partial S)_{V}$, are twin casualties of these thermostat definitions. None of the reversible 
deterministic thermostats provides a nonequilibrium analog for the equilibrium Gibbs entropy. The nonequilibrium Gibbs entropy diverges!

Despite this lack of a nonequilibrium entropy the incorporation of heat flow through the time-reversible thermostat forces leads to a microscopic understanding of the macroscopic Second Law of Thermodynamics9. We will see that this understanding involves Mandelbrot's fractals and the Lyapunov's instability spectrum. The irreversibility can occur in few-body systems. Even one-body Brownian motion can be treated in this way8.

\section{Computational Advances:}

In the 1950's Alder, Wainwright, and Wood used the computers at Livermore and Los Alamos to show that a few dozen hard spheres could characterize both solid and fluid phases. Alder and Wainwright also showed that, apart from fluctuations, the evolution of unusual initial states is described by the Boltzmann Equation ${ }^{10}$.

At both weapons laboratories, high-explosive work spawned an active interest in shockwaves. By 1967, the year of Lorenz' seminal work, hard spheres were passé. Vineyard, Rahman, and Verlet were successfully extending molecular dynamics to smooth pair potentials ${ }^{11}$. At Livermore I tried to use Rahman's ideas to make movies of shock-induced soft-sphere melting12. The movie project ultimately failed for lack of a reliable data storage device.

But times change. At Livermore we progressed through seven successive incarnations of CDC and CRAY computers, each more powerful than its predecessors. Now these once-remarkable supercomputers are dinosaurs, giving way to machines like Tony De Groot's SPRINT, which is 100 times more cost-effective. Now we can 
follow and display the motion of millions of atoms on a university budget ${ }^{13}$. The size and time scales of such simulations are approaching those of real microscale and nanoscale experiments. Another four or five orders of magnitude improvement 8 forecast in the near future ${ }^{14}$.

Simulation algorithms are changing too. In the 1950's solving Newton's equations of motion for a few dozen hard spheres was a challenge. In 1960 Vineyard was the first to formulate interesting nonequilibrium boundary conditions for particles with continuous forces 11 . Today, we can treat far from equilibrium flows with a million atoms using realistic interatomic forces. Boundary, constraint, and driving forces, added to the usual atomistic forces, furnish the sources of mass, momentum, and energy crucial to most nonequilibrium flows:

$$
\dot{p} \equiv F_{A}(q)+F_{B}(q)+F_{C}(q, p)+F_{D}(q, p) \equiv m\left[q_{t+d t}-q_{t}+q_{t-d t}\right] / d t^{2}
$$

These new motion equations are still deterministic and still timereversible. But they are not symplectic, so that phase volume can vary with time and exhibit irreversible behavior. Nevertheless the solution algorithms are based on Störmer's ideas from nearly a century ago. As computer capacity continues to expand, calculations incorporating electronic, as well as atomic, coordinates will become commonplace.

\section{Nonequilibrium Molecular Dynamics Develops:}

During the ten years leading up to Howard Hanley's 1982 Boulder conference on Nonlinear Fluid Behavior efficient algorithms consistent with the Green-Kubo relations were discovered for diffusion, shear and bulk viscosity, and heat conductivity ${ }^{15}$. Gauss' isokinetic thermostat was 
formulated as a differential equation with $F_{C} \equiv-\zeta p \equiv \dot{\Phi} p / 2 K . \quad[\Phi$ and $K$ are the potential and kinetic energies of the thermostatted degrees of freedom.] In 1982 it was not clear to outsiders that Green-Kubo linearresponse theory was an exact limiting case of the nonequilibrium simulations. Only specialists knew.

Nonequilibrium molecular dynamics was exposed to the scrutiny of the experts attending Howard Hanley's 1982 Boulder Conference, Nonlinear Fluid Behavior ${ }^{16}$. Discussion centered on the validity and reversibility of the motion equations, nonlinear response theory, the proper boundary conditions, and on the relation of calculations to properties of real molecules. By now these questions have been substantially resolved. For useful summaries see the reprint volume Simulation of Liquids and Solids 17 and the Proceedings of Michel Mareschal's Brussels meeting, Microscopic Simulations of Complex Flows ${ }^{18}$.

It has been confirmed that the finite-word-length accuracy of the simulations does not limit the validity of the results. Yorke, Yoshida, and others 19 have established the existence of "shadow trajectories" which lie close to computed ones. Yoshida's "shadow trajectories" are the most interesting. As a simple illustration of his general result, consider the smogth (q,p) trajectory generated by the "Yoshida Hamiltonian" $\mathrm{HY}=$ qpdt $+(q-p)^{2} / 2$. The trajectory approaches the harmonic oscillator one as $\mathrm{dt}$ is reduced. For finite dt the [Hamiltonian] trajectory is of course symplectic, conserving phase volume. But it also [surprisingly!] traces out the phasespace points generated by an algorithm equivalent to Stormer's:

$$
q_{t+d t}=q_{t}+p_{t} d t ; p_{t+d t}=p_{t}-q_{t+d t} d t
$$


Partly in response to discussions at Boulder I became convinced that the study of small systems was necessary to an understanding of nonequilibrium systems. Gary Morriss and I reported on some of these results at the Enrico Fermi Summer School at Lake Como in 198520. The small-system and time-reversibility studies both showed that Gibbs' entropy diverges for nonequilibrium steady states!

I continued working on small systems while on sabbatical in Vienna, working with Karl Kratky and Harald Posch while corresponding with Denis Evans, Brad Holian, and Gary Morriss. I became convinced that the Kawasaki-Visscher-Evans-Holian-Morriss exact but formal response theory 15 had conceptual problems when applied to nonequilibrium steady states. The equations for the phase-space distribution function diverged. A key consequence of the divergence was that Gibbs' statistical definition of temperature in terms of the phase-space entropy $S \equiv-k<\ln f>, T \equiv$

$(\partial E / \partial S)_{V}$, had to be abandoned. When the phase-space density $f(q, p, t)$ collapses onto a strange attractor Gibbs' entropy diverges. Thus temperature must be defined according to kinetic theory: $3 \mathrm{NkT} \equiv \Sigma \mathrm{p}^{2} / \mathrm{m}$.

A family of one-body "Galton Board" problems that I began to study with Tony Ladd in 1983 and followed up with Bill Moran ${ }^{21}$ showed that the fractals popularized by Mandelbrot generally underlie nonequilibrium systems and even some equilibrium ones.

We have studied several such few-body strange-attractor examples 21,22 . In every case the equations of motion were deterministic and time-reversible, and in every case Lyapunov instability broke the symmetry to provide irreversible behavior. Poincaré cross-sections cutting through five typical multifractal strange attractors are illustrated in Figure 2: 
1. Isokinetic Dissipative Motion in the Galton Board;

2. Field-Driven Conductivity in a Sinusoidal Potential;

3. Two-Body Shear Flow-a Shearing Galton Board;

4. One-dimensional, One-particle Thermodynamic PV Cycle;

5. Viscous Dissipative Motion in the Galton Board.

By 1987 the realization that time-reversible deterministic f nonequilibrium molecular dynamics always produces fractal structures led us to understand the Second Law of Thermodynznics as a time-symmetry breaking of Lyapunov-unstable thermostatto flows6,9. The examples in Figure 2 illustrate the general rule that, cespite time reversibility of the motion equations, the Lyapunov exponents, which give the averaged rate of expansion and contraction in phase space, always have a negative sum in steady nonequilibrium flows. For homogeneously thermostatted systems Sarman, Evans, and Morriss, have shown in addition that each such pair of exponents undergoes an equal negative shift 23 . The general case is more complicated. Figure 3 illustrates the shift of the spectran for an inhomogenous eight-body system, a shear flow thermostatted at the boundaries.

The predominantly negative Lyapunov exponents shrink the occupied phase-space, not only in volume, but also in dimensionality, well below the equilibrium value 24. A more complete quantitative understanding of the large-system dimensionality drop awaits the teraflop and petaflop machines of the next decade ${ }^{14}$. 


\section{Some Conclusions:}

From the pedagogical standpoint the main conceptual point revealed by analysing computer simulations is clear: Lyapunov's mechanical instability underlies Boltzmann's thermodynamic stability. Thus the microscopic sensitivity to initial conditions provides the averaging required for the inexorable work-to-heat dissipation associated with the Second Law of Thermodynamics. The macroscopic Second Law of Thermodynamics can be derived from the microscopic mechanical equations describing time-reversible deterministic thermostats. The NoséHoover thermostats fundamental to this derivation necessarily involve feedback. For a recent illustration of the Feedback concept, see Figure 4.

\section{Recent and Future Applications of Nonequilibrium Molecular Dynamics:}

Let us highlight a few recent examples of nonequilibrium flows and cite recent books for more6,15-18,20. Liem, Brown, and Clarke recently published very detailed density and temperature profiles for a nonequilibrium shear flow driven by isothermal boundaries 25 . Their profiles, reproduced in Figure 5, indicate the finite extent of boundary influences and the eventual convergence to a smooth hydrodynamic profile despite the huge gradients. The ordering and the low-density nonlinear transport coefficients in such flows have been simulated and analyzed by Hess and Loose 26 . Shockwave studies have continued, and show that linear transport theory is a good first approximation to this highly nonlinear problem. Klimenko and Dremin's shockwave simulations were brought up to date in 198027; these have now been followed by Robertson, Brenner, and White's simulations of the shock-induced dissociation of chlorine 28 . 
The Rayleigh-Bénard problem, discussed by Loren $\mathrm{z}^{4}$, has been the object of many simulations. Rapaport, Mareschal, and others have used molecular dynamics to generate intricate roll patterns which transfer heat between two reservoirs through convection 29 . I first saw the details of such patterns in Sitges, in 1980, where Gollub ${ }^{30}$ showed pictures of some laboratory rolls which had not yet stabilized on a timescale of 400 hours. These long times emphasize the limits of simulation and experiments.

The breaking of spatial symmetry in the Rayleigh-Bénard problem has solid-state analogs. Our indentation simulations, starting out with a perfectly symmetric single crystal, show the interesting loss of space symmetry ${ }^{13}$ shown in Figure 6. Grain growth studies, based on Holian's ideas ${ }^{13}$ for generating polycrystalline initial conditions, and Abraham's seminal work on spinodal decomposition ${ }^{31}$ also suggest the generality of symmetry breaking. Just as in the breaking of time symmetry the fundamental mechanism is deterministic chaos, Lyapunov instability.

\section{Extending Nonequilibrium Molecular Dynamics:}

Applications demand more practical work in the direction of simulating metals and covalent materials. Landman's pictures of recording head lubrication are a recent example 32 directly related to practical applications. This practical emphasis will grow. For realism the electrons must be included. There is much to be done with the new ideas for electronic motion simulation begun by Car and Parrinello33.

Nonequilibrium simulation has its limits. From an atomic prspective, a micron is a long distance and a microsecond is a long time/. There is a pressing need for extending the scope in time and space. There are many ways to try to do this. They need to be tried out and evaluated. 
Unfortunately these methods are fully as time-consuming as is the solution of the partial differential equations of continuum mechanics. One promising approach is to consider the interaction of continuum zones with particle-filled zones 13. Another is to use smooth-particle hydrodynamics 34 .

\section{Acknowledgment:}

I appreciate the organizers encouragement and the opportunity to speak here, The work which I have described involves the efforts of many colleagues, among them Tony De Groot, Brad Holian, my wife Carol Hoover, Jeff.Kallman, Tony Ladd, Susanne Lee, Bill Moran, Harald Posch, and Ered Wooten Harald kindly commented on a draft of this work. This presentation was in part supported by United States Department of Energy at the Lawrence Livermore National Laboratory under Contract W-7405-Eng-48 and in part supported by the Academy of Applied Science of Concord, New Hampshire.

\section{Figure Captions}

Figure 1. Our founding fathers, Boltzmann and Lyapunov.

Figure 2. Five deterministic time-reversible nonequilibrium strange attractors stabilized by Nosé-Hoover thermostats.

Figure 3. Equilibrium and Nonequilibrium Lyapunov spectra for a boundary-driven eight-atom shear flow.

Figure 4. Lee Lorenz' 25 May 1992 New Yorker drawing.

Figure 5. Density and temperature profiles for plane Couette flow.

Figure 6. Spatial symmetry loss during plane-strain indentation 


\section{References:}

1. R. Zwanzig, "Time Correlation Functions and Transport Coefficients in Statistical Mechanics", Annual Review of Physical Chemistry 16, 67 (1965); "Where Do We Go from Here?", Perspectives in Statistical Physics, H. J. Raveché, ed. (North-Holland, Amsterdam, 1981).

2. R. Kubo, "Opening Address to Oji Seminar on Nonlinear Nonequilibrium Statistical Mechanics", Progress of Theoretical Physics Supplement 64, 1 (1978).

3. A. W. Lees and S. F. Edwards, "The Computer Study of Transport Processes under Extreme Conditions", Journal of Physics C 5, 1921 (1972); E. M. Gosling, I. R. McDonald, and K. Singer, "On the Calculation by Molecular Dynamics of the Shear Viscosity of a Simple Fluid", Molecular Physics 26, 1475 (1973); L. V. Woodcock, "Isothermal Molecular Dynamics Calculations for Liquid Salts", Chemical Physics Letters 10, 257 (1971); W. T. Ashurst and W. G. Hoover, "Argon Shear Viscosity via a Lennard-Jones Potential with Equilibrium and Nonequilibrium Molecular Dynamics", Physical Review Letters 31, 206 (1973).

4. E. H. Lorenz, "Dimension of Weather and Climate Attractors", Nature 353, 241 (1991).

5. J. Gleick, Chaos (Viking, New York, 1987).

6. For general background, consult W. G. Hoover, Computational Statistical Mechanics (Elsevier, Amsterdam, 1991).

7. S. Nosé, "Unified Formulation of the Constant Temperature Molecular Dynamics Methods", Journal of Chemical Physics 81, 511 (1984).

8. D. Kusnezov, A. Bulgac, and W. Bauer, "Canonical Ensembles from Chaos", Annals of Physics 204, 155 (1990) and 214, 180 (1992).

9. B. L. Holian, W. G. Hoover, and H. A. Posch, "Resolution of Loschmidt's 
Paradox: The Origin of Irreversible Behavior in Reversible Atomistic Dynamics", Physical Review Letters 59, 10 (1987).

10. B. J. Alder and T. Wainwright, "Molecular Dynamics by Electronic Computers", in Transport Processes in Statistical Mechanics, Proceedings of the IUPAP Symposium, Brussels, 1956 (Interscience, NY, 1958), page 97. 11. J. B. Gibson, A. N. Goland, M. Milgram, and G. H. Vineyard, "Dynamics of Radiation Damage", Physical Review 120, 1229(1960); A. Rahman, "Correlation in the Motion of Atoms in Liquid Argon", Physical Review A 136, 405 (1964); L. Verlet, “Computer Experiments on Classical Fluids", Physical Review 159, 98 (1967).

12. R. E. Duff, W. H. Gust, E. B. Royce, A. C. Mitchell, R. N. Keeler, and W. G. Hoover, "Shockwave Studies in Condensed Media", Proceedings of the IUTAM Symposium on Behavior of Dense Media under High Dynamic Pressures (Paris, 1967), page 404.

13. W. G. Hoover, A. J. De Groot, and C. G. Hoover, "Massively Parallel Computer Simulation of Plane-Strain Elastic-Plastic Flow via Nonequilibrium Molecular Dynamics and Lagrangian Continuum Mechanics", Computers in Physics 6, 155 and cover (1992). 14. R. Preston, "The Mountains of Pi", The New Yorker, page 36, 2 March 1992.

15. D. J. Evans and G. P. Morriss, Nonequilibrium Liquids (Academic, New York, 1990).

16. Proceedings of the 1982 Boulder, Colorado Conference: "Nonlinear Fluid Behavior", Physica 118A, 1 (1983).

17. G. Ciccotti, D. Frenkel, and I. R. McDonald, eds. Simulation of Liquids and Solids (North-Holland, Amsterdam, 1987).

18. Microscopic Simulations of Complex Flows, M. Mareschal, Ed. 
(Plenum, New York, 1990).

19. C. Grebogi, S. M. Hammel, J. A. Yorke, and T. Sauer, "Shadowing of

Physical Trajectories in Chaotic Dynamics-Containment and Refinement", Physical Review Letters 65, 1527 (1990). H. Yoshida, "Construction of Higher Order Symplectic Integrators", Physics Letters A 150, 262 (1990). 20. Molecular Dynamics Simulation of Statistical Mechanics Systems, Proceedings of the 1985 Enrico Fermi Summer School at Varenna, G. Ciccotti and W. G. Hoover, eds. (North-Holland, Amsterdam, 1986). 21. W. G. Hoover and B. Moran, "Viscous Attractor for the Galton Board", Chaos (to appear); W. G. Hoover, H. A. Posch, B. L. Holian, M. J. Gillan, M. Mareschal, and C. Massobrio, "Dissipative Irreversibility from Nosé's Reversible Mechanics", Molecular Simulation 1, 79 (1987)..

22. W. G. Hoover, C. G. Hoover, W. J. Evans, B. Moran, J. A. Levatin, and E. A. Craig, "Irreversible Processes from Reversible Mechanics", in Reference 18.

23. S. Sarman, D. J. Evans, and G. P. Morriss, “Conjugate Pairing Rule and Thermal Transport Coefficients", Physical Review A 45, 2233 (1992). 24. H. A. Posch and W. G. Hoover, "Nonequilibrium Molecular Dynamics of Classical Fluids", in New Perspectives in Physics and Chemistry, J. J. C. Teixera-Dios (ed.), (Kluwer Academic Publishers, 1992, in press).

25. S. Y. Liem, D. Brown, and J. H. R. Clarke, "Investigation of the Homogeneous Shear Nonequilibrium Molecular Dynamics Method", Physical Review A 45, 3706 (1992).

26. W. Loose and S. Hess, "Anisotropy in Velocity Space Induced by Transport Processes", Physica A 174, 47 (1991); "Shear-Induced Ordering Revisited", in Reference 18.

27. B. L. Holian, W. G. Hoover, B. Moran, and G. K. Straub, "Shockwave 

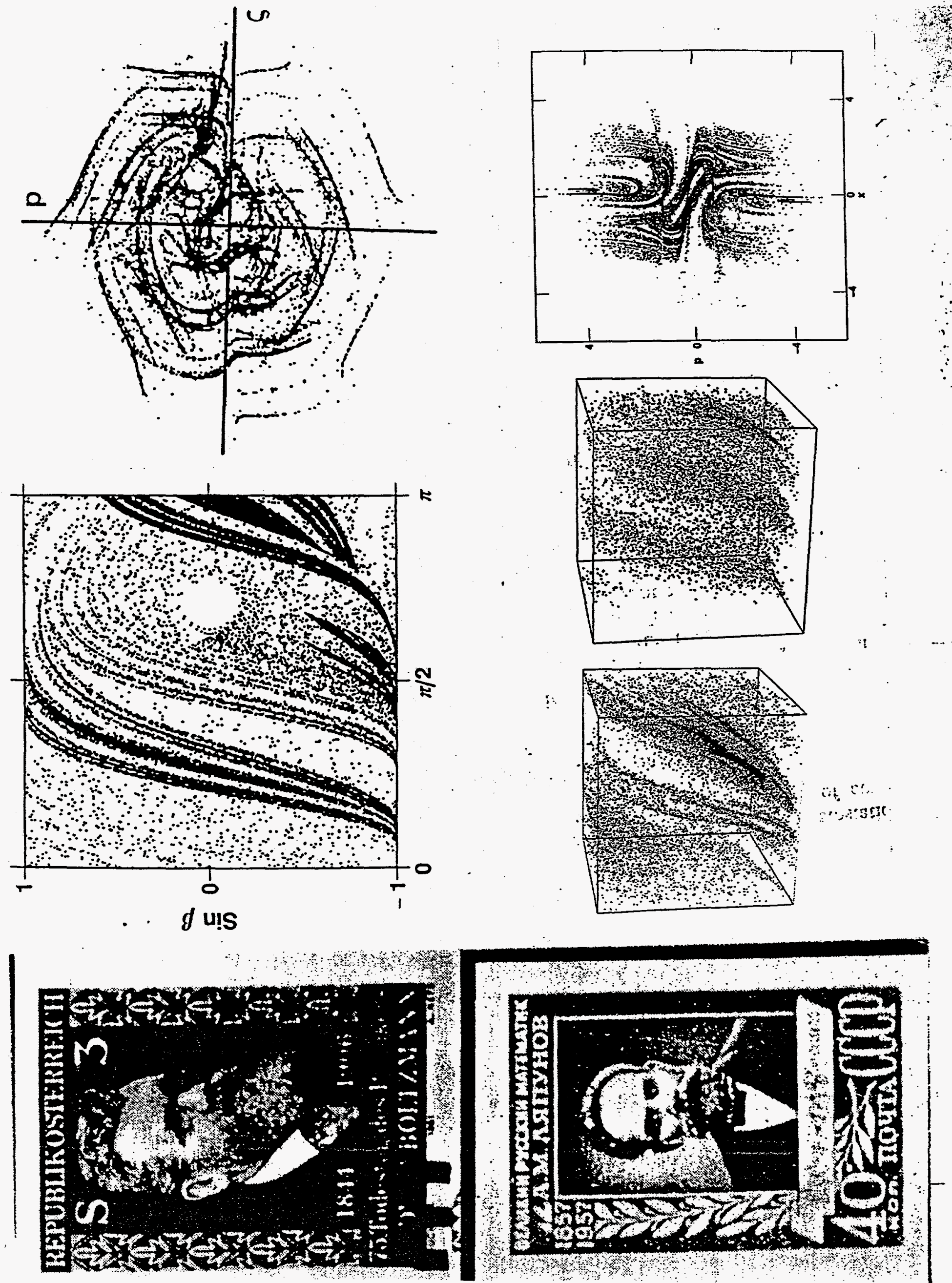

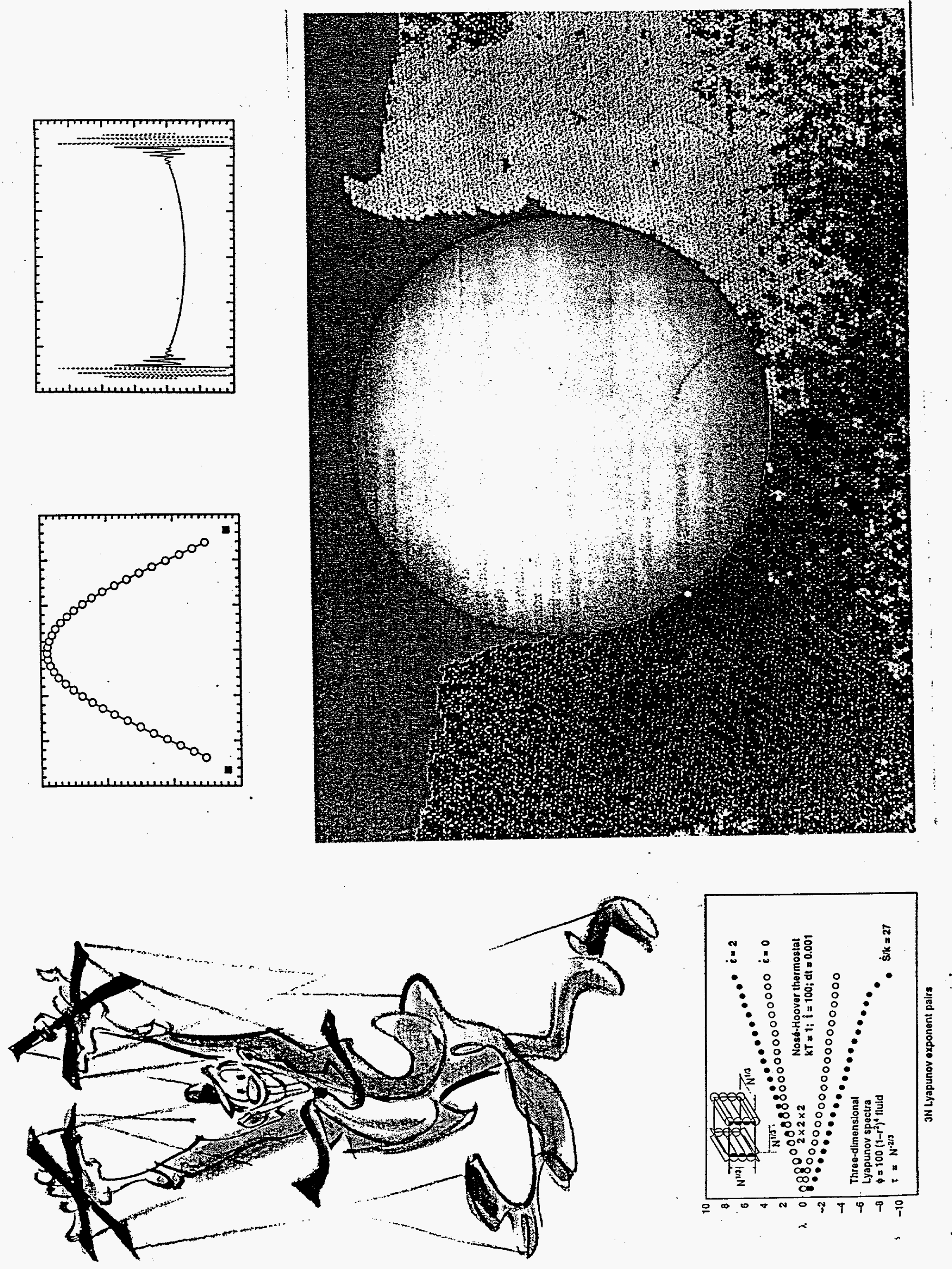